\title{
Prevalence of erectile dysfunction and associated factors among men with diabetes mellitus, Northern Ethiopia, 2020
}

\author{
Gebreegziabhier $\mathrm{G}^{1 *}$, Mekonnen $\mathrm{MS}^{2}$, Wtsadik $\mathrm{DA}^{3}$ and Nigus $\mathrm{G}^{4}$ \\ ${ }^{1}$ Department of Psychiatry, College of Medicine and Health Science, Aksum University, Aksum, Ethiopia \\ ${ }^{2}$ Department of Nursing, College of Health Science and Medicine, Dilla University, Dilla, Ethiopia \\ ${ }^{3}$ Department of Nursing, College of Health Science, Debre Berhan University, Debre Berhan, Ethiopia \\ ${ }^{4}$ School of Nursing, College of Health Science, Mekelle University, Mekelle, Ethiopia
}

\begin{abstract}
Background: Diabetes mellitus is the predominant risk factor for erectile dysfunction due to vascular and neurological complications. It affects the patient's quality of life by imposing psychological and emotional consequences including depression, anxiety, low self-esteem, and lack of self-confidence. Hence, this study aimed to scrutinize the prevalence of impotence and its associated factors among diabetic patients.

Methods: Institutional based cross-sectional study was employed on 330 systematically selected male diabetic patients in tertiary hospitals, Northern Ethiopia from March 2019 to January 2020. Data were collected using a structured questionnaire through face-to-face interviews, entered to Epi data version 4.4.2.1, and exported to SPSS version 25 for analysis. Binary logistic regression was used for analysis. Variables with $p<0.25$ in the bi-variable analysis were fitted to multivariate analysis. Then, the adjusted odds ratio with $95 \% \mathrm{CI}$ was used to report the association whereas statistical significances declared at $\mathrm{P} \leq 0.05$. Finally, the findings were presented using texts and frequency tables.

Results: This study was conducted on 330 male diabetic patients with an overall response rate of $97.1 \%$. The prevalence of erectile dysfunction among men with diabetes mellitus was found to be $87 \%$. Having average monthly income above poverty line [AOR=5.6; 95\%CI: 2.08-15.08], long duration on diabetic follow-up [AHR=1.67; 95\% CI: 1.40-2.00], not engaging in daily physical exercise [AOR=4.73; 95\%CI: 1.83-12.23], and using oral medications for diabetes mellitus [AHR= 6.27; 95\% CI: 2.01-19.58] were significantly associated with erectile dysfunction among male diabetic patients.

Conclusion: Erectile dysfunction is highly prevalent among diabetic patients. Moreover, prolonged diabetic follow-up, family monthly-income above the poverty line, using oral hypoglycemic agents, and lack of daily physical exercise were independent risk factors of impotence. Thus, it is advisable to conduct universal screening for this population group with open discussion during diabetic visits to enable timely detection and management.
\end{abstract}

\section{Background}

Diabetes mellitus is a chronic metabolic disorder which is characterized by hyperglycemia caused by a defect in insulin action, insulin secretion, or both. It is common and on rising disease worldwide and expected to affect more than half billion people by the year 2035 [1]. DM is associated with multiple complications of which sexual dysfunction in men is the one [2-4].

Erectile dysfunction (ED) can be defined as an inability to obtain and/or maintain an erection of the penis satisfactory for sexual intercourse. ED can result from several physiological and psychological conditions in which diabetes mellitus (DM) is an independent risk factor [5]. It is proposed that in patients with DM, vascular and neurological complications of the illness cause ED. It may be related to associated clinical conditions such as hypertension, cigarette smoking obesity, metabolic syndrome, and/or dyslipidemia.

ED is a highly frequent health problem occurring among 18.4$41.5 \%$ of men in the general population and negatively affects their quality of life [6,7]. It is a primary chief complaint concerning more than $50 \%$ of all men treated with sexual disorder [8]. The prevalence is even higher among diabetic patients varying from $14-98 \%$ [9-12].
Diabetic patients with erectile dysfunction encountered various psychological consequences including depression, anxiety, low selfesteem, and lack of self-confidence $[13,14]$ negatively influencing the patient's interaction with family and important others.

Both psychological and biological treatments are effective in managing ED among patients with DM. Even though treatments increased the level of sexual and life satisfaction, treatment-seeking behavior among men with ED is minimal [15-18]. Factors including but not limited to age, BMI, peripheral neuropathy, longer duration and mode of therapy had a significant association with ED [11,12].

Unlike the previous studies, this study incorporated patients with multiple complications since it was conducted on tertiary care centres.

*Correspondence to: Gebreslassie Gebreegziabhier, Department of Psychiatry, College of Medicine and Health Science, Aksum University, Aksum, Ethiopia, E-mail: ggebreegziabhier@gmail.com

Key words: erectile dysfunction, impotence, diabetes, Ethiopia, prevalence, factors Received: June 10, 2020; Accepted: June 19, 2020; Published: June 25, 2020 
Differently, this study also investigated the possible effects of important factors such as depression, anxiety, and other diabetic complications on ED. Therefore, this study aimed to assess the magnitude of ED and its associated factors among patients with diabetes mellitus at tertiary hospitals, Ethiopia.

\section{Methods and materials}

\section{Study design, area, and period}

An institutional-based cross-sectional study employed from March 2019 to January 2020 among diabetic patients who had follow-up at diabetic clinics of tertiary care centres in Tigray, Ethiopia. There are two tertiary care centers throughout the region: Ayder and Aksum Comprehensive Specialized hospitals serving for an overall population of more than 5,377,144 [19]. Ayder comprehensive specialized hospital serve for Tigray, Afar, Southeastern parts of the Amhara region population as well as the Eritrean refugees whereas, patients who came from central, western and northwest parts of Tigray regional state served by Aksum referral hospital. The diabetic clinic at Ayder has four regular working days within a week.

\section{Population of the study}

All male patients diagnosed with diabetes mellitus and had followup at diabetic clinics of tertiary hospitals in Tigray. Patients greater than or equal to 18 years of age and who engaged in sexual activity over the past six months were included in the study whereas those who missed their regular follow-up visits and severely ill were not incorporated.

\section{Sample size determination and sampling procedure}

The sample size was calculated by using single population proportion formula $\left(\mathrm{n}=\left(\mathrm{z}^{\wedge} 2 \mathrm{pq}\right) / \mathrm{d}^{\wedge} 2\right)$ by considering the following assumptions: prevalence of $\mathrm{ED}=69.9 \%$ from the previous study conducted in central $\&$ north Tigray [16], 95\% level of confidence $(\mathrm{z} \alpha / 2=1.96), 5 \%$ degree of freedom $(\mathrm{d}=0.05)$.

$$
\left.\mathrm{n}=\left((1.96)^{\wedge} 2[0.699)^{\star}(1-0.699)\right]\right) /[(0.05)]^{\wedge} 223.3
$$

By adding a $5 \%$ non-response rate, the required sample size to conduct this study was 340 . Then, 340 participants were selected systematically by incorporating every other patient after adjusting in order of their arrival (service turn). Sampling interval (k) was determined by dividing the total registered one-month diabetic appointments (taken from the patient's appointment chart) during the data collection period by the estimated sample size. There were 817 scheduled appointments from March 15 to April 15, 2019; of those 234 had repeated follow-up schedules in the specified period. Thus, after excluding repeated appointments, we had 700 diabetic visits that yield $\mathrm{k}=2$ (interval) when divided by 340 .

\section{Operational definition}

Erectile dysfunction was declared if patients correctly responded $\leq$ to 21 out of 25 erectile function assessment questions that are the international index of erectile function (IIEF- 5) [20]. Patients diagnosed with ED were classified as having mild, moderate, and severe ED if they correctly responded 1-7, 8-11, and 12-21 questions respectively. Social support refers to support needed at times when individuals suffered difficulties and critical conditions like financial, social, and psychological crisis and assessed by the Oslo-3 scales which has a total of 14 scores \& classified into three broad categories such that: Poor support $=3-8$ scores, moderate support $=9-11$ scores and, strong support $=12-14$ scores [21]. Physically active was when subjects engaged in moderate activities at least 5 times per week, duration 30 minutes, and/or vigorous activities at least 3 times per week, duration 20 minutes. Current substance use was using at least one of a specific substance (alcohol, chat, tobacco, others) for non-medical purposes within the last three months whereas ever use of substance indicates using at least one of any of these specific substances for the non-medical purpose at least once in a lifetime. Physical illness: those respondents who responded to having a chronic physical illness that was diagnosed before from any private and public health institution and currently on follow up. Depression was assessed using HADS-D criteria and those who scored $<=10$ from HADS-D were considered as having depression [22].

\section{Data collection tools and procedures}

Data were collected by face-to-face interview, chart review, and physical examination using a structured interviewer-administered questionnaire. The questionnaire comprised of the socio-demographic questionnaire, substance-use assessment tools, questions and measurements for clinical factors, social support assessment questions (Oslo-3 scale), depression (HADS_D), questions for acceptance, and use of treatment for sexual problem, and IIEF (to assess erectile dysfunction). The reliability of IIEF was calculated and found to have Cronbach's $\alpha$ for the total IIEF score $\alpha=0.89$ with each domain Cronbach's $\alpha=$ erectile dysfunction $=0.86$ [23]. The patients FBS and BMI were measured at the time of data collection. Height (in meters) was taken using the hospital height scale while the patient is standing upright and weight (in kilograms) was measured using a standard weighing. The body mass index (BMI) was derived by dividing the weight $(\mathrm{kg})$ by the square of the height $(\mathrm{m})$ and the classification of obesity was done according to the World Health Organization [24]. The data collection period was from March 15 to April 15, 2019.

\section{Data quality control}

The questionnaire was developed in English, translated into local languages (Tigrigna and Amharic) by language experts, and translated back to English for consistency and understandability of the tool. The questionnaire was also pretested on 5\% (22 subjects) of samples at Mekelle hospital diabetic clinic which were not incorporated in the actual data for analysis. Four data collectors (male BSc nurses) and two supervisors (psychiatric nurses) were and trained for one day on the requirements and methods of data collection. Data were evaluated and crosschecked for completeness daily.

\section{Data processing, analysis, interpretation, and presentation}

Data were cleaned, coded, and entered using Epi data version 4.4.2.1 and it was exported to SPSS version 25 for analysis. Cross-tabulation was done for the exploration of the data, to clear missing values, and to determine the number of expected events per cell. Continuous variables were described using mean with standard deviation and median with IQR based on its applicability whereas relative frequency and percent used for categorical variables. Binary logistic regression was used for the analysis of the data. Multi-collinearity was checked using variance inflation factor (mean vif $=1.69$ ) and model fitness was checked using Hosmer and Lemeshow test $(\mathrm{p}=0.223)$. Variables with $\mathrm{p}$-values $<0.25$ in the bi-variable analysis were transferred to the multivariate analysis to identify the independent effects of each variable to the outcome variable. An adjusted odds ratio with $95 \%$ confidence intervals was computed to identify the presence and strength of associations, and statistical significance was declared at $\mathrm{p}<0.05$. Finally, the result was presented in the form of plain texts and frequency tables. 


\section{Ethical consideration}

Ethical clearance was obtained from Aksum university, college of health sciences ethical Review committee. Then officials at different levels of the selected hospitals had been communicated about the purpose of the study. Written Informed consent was received from the respondents (patients) after explaining the details of the study and confirmation of their willingness. Those who were not willing to participate in the study were not forced to participate and were notified that they have the right to refuse or terminate the interview at any time. Confidentiality of the information was secured throughout the study process by using code instead of any personal identifier \& is meant only for the study.

\section{Results}

\section{Sociodemographic characteristics}

An overall 330 male study subjects having sexual engagement in the past six months who had diabetic follow-up at diabetic clinics of tertiary care hospitals of Tigray region participated in this study with an overall response rate of $97.1 \%$.

According to this study, the median age of the participants was 40 years $(\mathrm{IQR}=30)$ ranging from $21-73$ years with $90 \%$ of subjects aged less than 65 years. The proportion of erectile dysfunction among participants aged above 65 years was $89.8 \%$ with a narrow difference among those aged below 34 years $(87.2 \%)$. The majority of participants were orthodox religious followers 300 (90.9\%) followed by Muslims $(8.8 \%)$ while 123 (37.3\%) were merchants. Regarding the marriage status, the highest proportions $(72.7 \%)$ of study subjects were married and the lowest (2.7\%) were widowed. About $43 \%$ attended school up to college-level or above, of which $84.5 \%$ experienced erectile dysfunction whereas about $97.8 \%$ of the participants who never attended school had erectile dysfunction (Table 1).

One-hundred-three (31.2\%) patients had a family monthly income below the poverty line ( $<1.9$ pounds/day) and the median monthly income was 68 pounds $(\mathrm{IQR}=99)$ with $25 \%$ of participants paid below 34.1 pounds.

The proportion of erectile dysfunction among patients diagnosed with type I and type II diabetes mellitus was $89 \%$ \& $80.5 \%$ respectively. Besides, one-fourth of the participants had a fasting blood sugar level of less than $110 \mathrm{~g} / \mathrm{dl}$ and about $215(65.2 \%)$ had FBS of $126 \mathrm{~g} / \mathrm{dl}$ or above, of those 183 (85.1\%) experienced erectile dysfunction. The average BMI among study participants was recorded as $22.9 \mathrm{Kg} / \mathrm{m} 2$ $(\mathrm{SD}=3.8)$. Forty-nine (14.8\%), 85 (25.8\%), and 9 (2.7\%) study subjects were underweight, overweight, and obese with respective proportion of erectile dysfunction of 36 (73.5\%), 78 (91.8\%), and 7 (77.8\%) among each category. One-hundred-sixteen (90.6\%) of 128 participants having co-morbidities (hypertension, cardiac and kidney insufficiency), and $51(83.6 \%)$ of 61 diagnosed with diabetic complications (retinopathy, neuropathy, foot ulcer, and others) were found to have erectile dysfunction (Table 2).

On the other hand, 65 (19.7\%) had a history of erectile dysfunction of which about 33 used medications for the problem. Among the total 330 study subjects included, only $50(15.2 \%)$ thought medication would help for erectile dysfunction. About 219 (66.4\%) of patients had diabetic follow-up for more than five years at diabetic clinics. The median diabetic follow-up among the participants was 6.5 years (IQR=6) with $75 \%$ of patients had followed for a maximum of nine years. The proportion of patients who developed erectile dysfunction was higher (93.2\%) among patients with prolonged follow-up (>=5years) than those followed for less than five years (74.8\%) (Table 2).

The study finding also revealed that about 27 (8.2\%), 238 (72.1\%), and $27(8.2 \%)$ had a history of use of chat, alcohol, and cigarette respectively while $41(12.4 \%), 101(30.6 \%)$, and $25(7.6 \%)$ participants have been using chat, alcohol, and cigarette at the time of data collection in that order. Concerning the daily physical exercise, 181 (54.8\%) did not perform the daily physical exercise of which 165 (91.2\%) faced erectile dysfunction. Furthermore, 136 (41.2\%) participants thought

Table 1. Distribution of socio-demographic characteristics among men diagnosed with diabetes mellitus who had follow-up at diabetic clinics of tertiary hospitals: Northern Ethiopia: 2020 $(\mathrm{N}=330)$

\begin{tabular}{|c|c|c|c|c|c|c|}
\hline \multirow{3}{*}{$\begin{array}{c}\text { Independent } \\
\text { Variables }\end{array}$} & \multirow{3}{*}{ Categories } & \multicolumn{5}{|c|}{ Erectile dysfunction } \\
\hline & & \multicolumn{2}{|c|}{ Yes } & \multicolumn{2}{|c|}{ No } & \multirow{2}{*}{ Total (\%) } \\
\hline & & Observed (\%) & Expected & Observed (\%) & Expected & \\
\hline \multirow{2}{*}{ Ethnicity } & Tigray & $286(88.3)$ & 281.8 & $38(11.7)$ & 42.2 & $324(98.2)$ \\
\hline & Others & $1(16.7)$ & 5.2 & $5(83.3)$ & 0.8 & $6(1.8)$ \\
\hline \multirow{3}{*}{ Religion } & Orthodox & $262(87.3)$ & 260.9 & $38(12.7)$ & 39.1 & $300(90.9)$ \\
\hline & Muslim & $24(82.8)$ & 25.2 & $5(17.2)$ & 3.8 & $29(8.8)$ \\
\hline & Protestant & $1(100)$ & 0.9 & 0 & 0.1 & $1(0.3)$ \\
\hline \multirow{4}{*}{ Marital status } & Married & $209(87.1)$ & 208.7 & $31(12.9)$ & 31.3 & $240(72.73)$ \\
\hline & Divorced & $31(91.2)$ & 29.6 & $3(8.8)$ & 4.4 & $34(10.3)$ \\
\hline & Widowed & $9(100)$ & 7.8 & 0 & 1.2 & $9(2.73)$ \\
\hline & Single & $38(80.9)$ & 40.9 & $9(19.1)$ & 6.1 & $47(14.24)$ \\
\hline \multirow{4}{*}{ Educational status } & Illiterate & $44(97.8)$ & 39.1 & $1(2.2)$ & 5.9 & $45(13.6)$ \\
\hline & Primary & $101(93.5)$ & 93.9 & $7(6.5)$ & 14.1 & $108(32.7)$ \\
\hline & Secondary & $22(62.9)$ & 30.4 & $13(37.1)$ & 4.6 & $35(10.6)$ \\
\hline & Collage \& above & $120(84.5)$ & 123.5 & $22(15.5)$ & 18.5 & $142(43.1)$ \\
\hline \multirow{4}{*}{ Occupation } & Merchant & $92(74.8)$ & 107.0 & $31(25.2)$ & 16.0 & $123(37.3)$ \\
\hline & Home & $38(90.5)$ & 36.5 & $4(9.5)$ & 5.5 & $42(12.7)$ \\
\hline & Servant & $108(95.6)$ & 98.3 & $5(4.4)$ & 14.7 & $113(34.2)$ \\
\hline & Other & $49(94.2)$ & 45.2 & $3(5.8)$ & 6.8 & $52(15.8)$ \\
\hline \multirow{2}{*}{ Family monthly income } & $\begin{array}{c}\text { Below } \\
\text { poverty line }\end{array}$ & $86(83.5)$ & 89.6 & $17(16.5)$ & 13.4 & $103(31.2)$ \\
\hline & $\begin{array}{c}\text { Above } \\
\text { poverty line }\end{array}$ & $201(88.5)$ & 197.4 & $26(11.5)$ & 29.6 & $227(68.8)$ \\
\hline
\end{tabular}


that counseling would not help for erectile dysfunction. Only $33(10 \%)$ of study subjects got strong (recommended) social support and $90.6 \%$ of patients among those who received poor social support had faced erectile dysfunction. Moreover, 77 (23.3\%) patients had anxiety and $81(24.5 \%)$ of them developed depression. About $89.6 \%$ of those with anxiety had erectile dysfunction whereas more than $90 \%$ of patients with depression experienced erectile dysfunction (Table 3).

Consequently, 287 (87\%) participants experienced erectile dysfunction whereas 43 (13\%) did not. Of those having erectile

Table 2. Distribution of clinical characteristics among men diagnosed with diabetes mellitus who had been attending at diabetic clinics of tertiary hospitals: Northern Ethiopia: 2020 (N=330)

\begin{tabular}{|c|c|c|c|c|c|c|}
\hline \multirow{3}{*}{$\begin{array}{l}\text { Independent } \\
\text { variables }\end{array}$} & \multirow{3}{*}{ Categories } & \multicolumn{5}{|c|}{ Erectile dysfunction } \\
\hline & & \multicolumn{2}{|c|}{ Yes } & \multicolumn{2}{|c|}{ No } & \multirow{2}{*}{ Total (\%) } \\
\hline & & Observed (\%) & Expected & Observed (\%) & Expected & \\
\hline \multirow{2}{*}{ Type of DM } & Type I & $62(80.5)$ & 67.0 & $15(19.5)$ & 10.0 & $77(23.3)$ \\
\hline & Type II & $225(88.9)$ & 220.0 & $28(11.1)$ & 33.0 & $253(76.7)$ \\
\hline \multirow{2}{*}{ FBS (mg/dl) } & $<126$ & $104(90.4)$ & 100.0 & $11(9.6)$ & 15.0 & $115(34.8)$ \\
\hline & $>=126$ & $183(85.1)$ & 187.0 & $32(14.9)$ & 28.0 & $215(65.2)$ \\
\hline \multirow{4}{*}{ BMI $\left(\mathrm{Kg} / \mathrm{m}^{2}\right)$} & Underweight & $36(73.5)$ & 42.6 & $13(26.5)$ & 6.4 & $49(14.8)$ \\
\hline & Normal & $166(88.8)$ & 162.6 & $21(11.2)$ & 24.4 & $187(56.7)$ \\
\hline & Overweight & $78(91.8)$ & 73.9 & $7(8.2)$ & 11.1 & $85(25.8)$ \\
\hline & Obesity & $7(77.8)$ & 7.8 & $2(22.2)$ & 1.2 & $9(2.7)$ \\
\hline \multirow{2}{*}{ DM drugs used } & Insulin & $129(83.2)$ & 134.8 & $26(16.8)$ & 20.2 & $155(47)$ \\
\hline & OHA & $158(90.3)$ & 152.2 & $17(9.7)$ & 22.8 & $175(53)$ \\
\hline \multirow{2}{*}{ Co-morbidities } & No & $171(84.7)$ & 175.7 & $31(15.3)$ & 26.3 & $202(61.2)$ \\
\hline & Yes & $116(90.6)$ & 111.3 & $12(9.4)$ & 16.7 & $128(38.8)$ \\
\hline \multirow{2}{*}{ DM Complications } & No & $236(87.7)$ & 233.9 & $33(12.3)$ & 35.1 & $269(81.5)$ \\
\hline & Yes & $51(83.6)$ & 53.1 & $10(16.4)$ & 7.9 & $61(18.5)$ \\
\hline \multirow{2}{*}{ History of ED } & Yes & $59(90.8)$ & 56.5 & $6(9.2)$ & 8.5 & $65(19.7)$ \\
\hline & No & $228(86)$ & 230.5 & $37(14)$ & 34.5 & $265(80.3)$ \\
\hline \multirow{2}{*}{ Ever used drugs for ED } & Yes & $25(75.8)$ & 28.7 & $8(24.2)$ & 4.3 & $33(10)$ \\
\hline & No & $262(88.2)$ & 258.3 & $35(11.8)$ & 38.7 & $297(90)$ \\
\hline \multirow{2}{*}{$\begin{array}{l}\text { Do you think medication } \\
\text { help for ED }\end{array}$} & Yes & $42(84)$ & 43.5 & $8(16)$ & 6.5 & $50(15.2)$ \\
\hline & No & $245(87.5)$ & 243.5 & $35(12.5)$ & 36.5 & $280(84.8)$ \\
\hline
\end{tabular}

Abbreviations: DM: diabetes mellitus; FBS: fasting blood sugar; mg/dl: milligrams per deciliter; BMI: body mass index; Kg/m²: kilograms per meter square; OHA: oral-hypoglycemic agents; ED: erectile dysfunction

Table 3. Distribution of behavioral and psychological factors among men diagnosed with diabetes mellitus who had been attending at diabetic clinics of tertiary hospitals: Northern Ethiopia: $2020(\mathrm{~N}=330)$

\begin{tabular}{|c|c|c|c|c|c|c|}
\hline \multirow{3}{*}{$\begin{array}{c}\text { Independent } \\
\text { Variables }\end{array}$} & \multirow{3}{*}{ Category } & \multicolumn{5}{|c|}{ Erectile dysfunction } \\
\hline & & \multicolumn{2}{|c|}{ Yes } & \multicolumn{2}{|c|}{ No } & \multirow{2}{*}{ Total (\%) } \\
\hline & & Observed (\%) & Expected & Observed (\%) & Expected & \\
\hline \multirow{2}{*}{ Ever used chat } & Yes & $17(63)$ & 23.5 & $10(37)$ & 3.5 & $27(8.2)$ \\
\hline & No & $270(89.1)$ & 263.5 & $33(10.9)$ & 39.5 & $303(91.8)$ \\
\hline \multirow{2}{*}{ Current chat use } & Yes & $29(70.7)$ & 35.7 & $12(29.3)$ & 5.3 & $41(12.4)$ \\
\hline & No & $258(89.3)$ & 251.3 & $31(10.7)$ & 37.7 & $289(87.6)$ \\
\hline \multirow{2}{*}{ Ever used alcohol } & Yes & $215(90.3)$ & 207.0 & $23(9.7)$ & 31.0 & $238(72.1)$ \\
\hline & No & $72(78.3)$ & 80.0 & $20(21.7)$ & 12.0 & $92(27.9)$ \\
\hline \multirow{2}{*}{ Current alcohol use } & Yes & $89(88.1)$ & 87.8 & $12(11.9)$ & 13.2 & $101(30.6)$ \\
\hline & No & $198(86.5)$ & 199.2 & $31(13.5)$ & 29.8 & $229(69.4)$ \\
\hline \multirow{2}{*}{ Ever used cigarette } & Yes & $16(59.3)$ & 23.5 & $11(40.7)$ & 3.5 & $27(8.2)$ \\
\hline & No & $271(89.4)$ & 263.5 & $32(10.6)$ & 39.5 & $303(91.8)$ \\
\hline \multirow{2}{*}{ Current cigarette use } & Yes & $14(56)$ & 21.7 & $11(44)$ & 3.3 & $25(7.6)$ \\
\hline & No & $273(89.5)$ & 265.3 & $32(10.5)$ & 39.7 & $305(92.4)$ \\
\hline \multirow{2}{*}{ Daily physical exercise } & Yes & $122(81.9)$ & 129.6 & $27(18.1)$ & 19.4 & $149(45.2)$ \\
\hline & No & $165(91.2)$ & 157.4 & $16(8.8)$ & 23.6 & $181(54.8)$ \\
\hline \multirow{2}{*}{$\begin{array}{l}\text { Do you think counseling } \\
\text { help for ED }\end{array}$} & Yes & $173(89.2)$ & 168.7 & $21(10.8)$ & 25.3 & $194(58.8)$ \\
\hline & No & $114(83.8)$ & 118.3 & $22(16.2)$ & 17.7 & $136(41.2)$ \\
\hline \multirow{3}{*}{ Social support } & Poor & $183(90.6)$ & 175.7 & $19(9.4)$ & 26.3 & $202(61.2)$ \\
\hline & Moderate & $83(87.4)$ & 82.6 & $12(12.6)$ & 12.4 & $95(28.8)$ \\
\hline & Strong & $21(63.6)$ & 28.7 & $12(36.7)$ & 4.3 & $33(10)$ \\
\hline \multirow{2}{*}{ Anxiety } & Yes & $69(89.6)$ & 67.0 & $8(10.4)$ & 10.0 & $77(23.3)$ \\
\hline & No & $218(86.2)$ & 220.0 & $35(13.8)$ & 33.0 & $253(76.7)$ \\
\hline \multirow{2}{*}{ Depression } & Yes & $73(90.1)$ & 70.4 & $8(9.9)$ & 10.6 & $81(24.5)$ \\
\hline & No & $214(85.9)$ & 216.6 & $35(14.1)$ & 32.4 & $249(75.5)$ \\
\hline
\end{tabular}

Abbreviation: ED: erectile dysfunction 
dysfunction, 75 (26.13\%) had severe dysfunction, 69 (24.04\%) had moderate, and 143 (49.83\%) had mild dysfunction.

\section{Associated factors of erectile dysfunction}

After the description of the data, the bi-variable analysis was conducted using the binary logistic regression model to determine the variables that should be fitted to the final model for multivariate analysis. Some variables such as religion, ethnicity, occupation, marital status, and educational status were left out of the final model since they have less than $20 \%$ predicted events per cell. Furthermore, ever and current use of cigarette were further excluded because of the collinearity effect.

Afterward, in the multivariate analysis, family monthly income, duration of diabetic follow-up, daily physical exercise, and DM medications used were significantly associated with erectile dysfunction among male diabetic patients (Table 4).

The multivariable analysis result showed that those DM patients who had average family monthly income above the poverty line were 5.6 times more likely to experience erectile dysfunction compared with those having monthly income below poverty line counterparts [AOR=5.6; 95\%CI: 2.08-15.08]. The odds of diabetic men who did not perform the daily physical exercise to acquire erectile dysfunction were 4.73 times higher than their counterparts $\operatorname{did}[\mathrm{AOR}=4.73 ; 95 \% \mathrm{CI}$ :
1.83-12.23]. The other factor that showed significant association was the duration of diabetic follow-up. Male patients who had diagnosed with DM and on diabetic follow-up for a long period were more likely to acquire erectile dysfunction. For each year increase in the duration of diabetic follow up, the odds of experiencing ED will increase by 1.67 [AHR=1.67; 95\% CI: 1.40-2.00]. Again, those patients who took oral hypoglycemic agents for DM management were also 6.27 times at an increased risk of experiencing ED compared to those who managed with insulin injection [AHR= 6.27; 95\% CI: 2.01-19.58] (Table 4).

\section{Discussion}

This study was aimed to determine the prevalence and associated factors of erectile dysfunction among male diabetic patients who had follow-up at diabetic clinics of tertiary care hospitals. An overall 330 study subjects who had diabetic follow-up at tertiary care hospitals and engaged to sexual intercourse in the past six months participated in this study with an overall response rate of $97.1 \%$.

The prevalence of erectile dysfunction among male patients with DM in this study was found to be $87 \%$ [95\% CI: 82.9-90.2]. Some of the previous study reports were in line with this finding [18,25]. However, this figure was higher than the global estimate of ED among DM men, $35-75 \%[26,27]$ and most of the previous study findings [16,28-33].

Table 4. Bi-variable and multivariate analysis for associated factors of erectile dysfunction among men diagnosed with diabetes mellitus who had been attending at diabetic clinics of tertiary hospitals: Northern Ethiopia: $2020(\mathrm{~N}=330)$

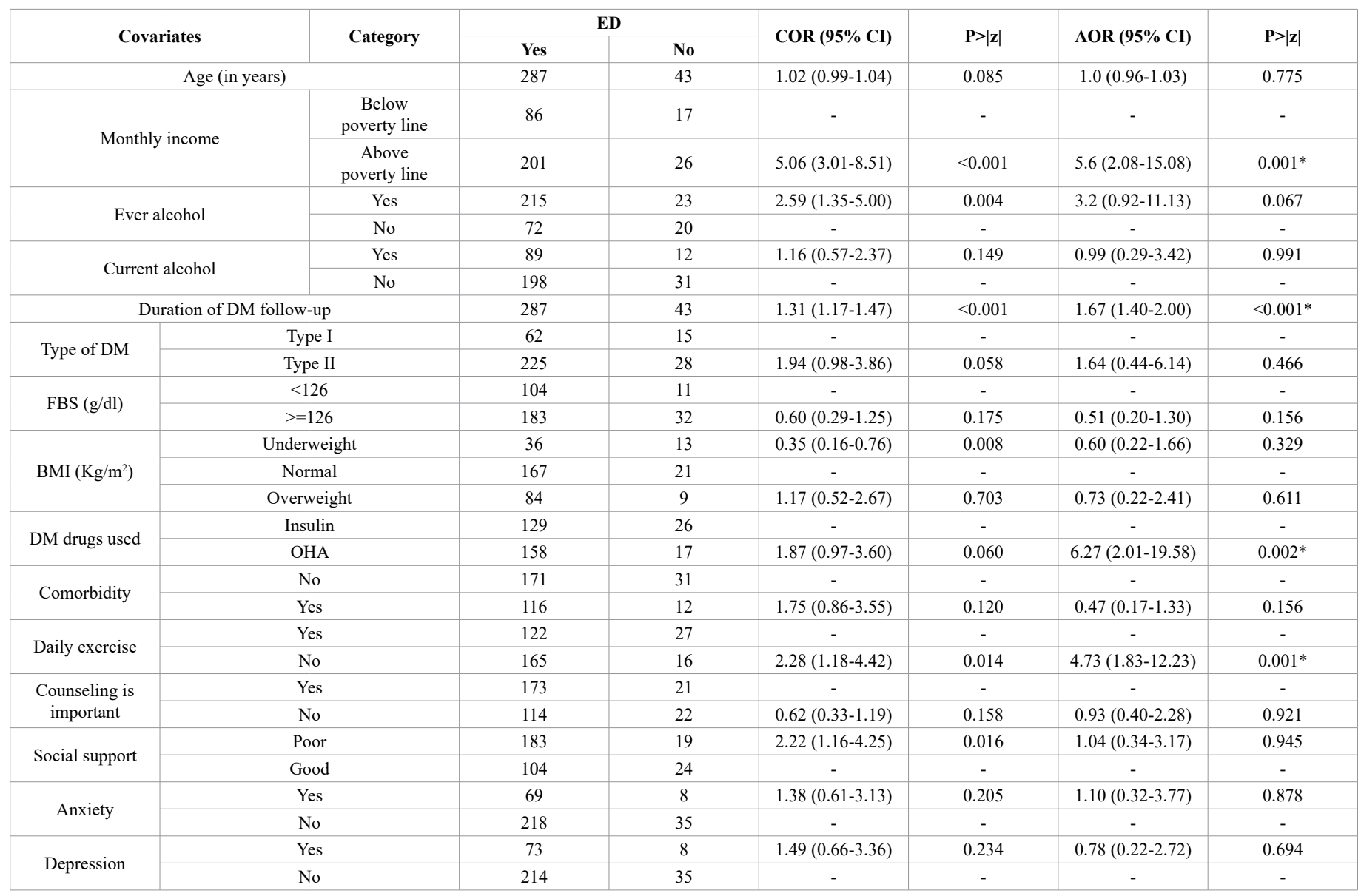

Note: *significant at $5 \%$ level of significance

Abbreviations: ED: erectile dysfunction; COR: crude odds ratio; AOR: adjusted odds ratio; CI: confidence interval; DM: diabetes mellitus; FBS: fasting blood sugar; mg/dl: milligrams per deciliter; BMI: body mass index; $\mathrm{Kg} / \mathrm{m}^{2}$ : kilograms per meter square; OHA: oral hypoglycemic agents 
This variation could be due to the possible difference in ED screening tendency among diabetic patients from setting to setting, the access and utilization of medications for ED, and diagnostic criteria used in those studies.

The multivariate logistic regression analysis showed that daily physical exercise, family monthly income, duration of diabetic followup, and DM medications used were significantly associated with erectile dysfunction among male diabetic patients in this study.

The odds of diabetic men who did not perform the daily physical exercise to acquire erectile dysfunction were 4.73 times higher than their counterparts did $[\mathrm{AOR}=4.73 ; 95 \% \mathrm{CI}$ : 1.83-12.23]. This finding was supported by the former study conducted at Mizan-Aman and Tepi towns [34]. Several explanations could be stated for this. In the case of diabetics, the elevated blood sugar level inhibits nitric oxide production, the chemical that allows penile tissue to relax, and increased penile blood flow resulting in an erection [35,36]. Physical exercise is one of the pillars of glycemic control measures while those who did not engage in daily physical exercise tend to have poorly controlled blood sugar levels. On the other hand, diabetes and obesity have undeniable pathophysiologic link-up. Physical exercise aids to have a balanced bodyweight and that in turn contributed to increased testosterone levels and enhanced blood flow. Besides, physical exercise is associated with stress reduction and relaxation activities that are significantly related to effective blood sugar level control [37].

This study also revealed that having an average monthly income above the poverty line was independently associated with erectile dysfunction among diabetic men. DM patients who had monthly income above the poverty line were 5.6 times more likely to experience erectile dysfunction compared with those having monthly income below poverty line counterparts [AOR=5.6; 95\%CI: 2.08-15.08]. Contradicting result was reported from a previous study done in northern Ethiopia [16] in which lower monthly income was identified as an independent risk factor for erectile dysfunction. This discrepancy might be because of the possible difference in the living standards of study subjects between these studies where most of the participants in the former study were farmers that involves relatively frequent physical activities compared to merchants and employers $(>81.5 \%)$ in the current study.

The other factor that significantly associated with erectile dysfunction was the duration of diabetic follow-up. Male patients who had diagnosed with DM and on diabetic follow-up for a long period were more likely to acquire erectile dysfunction. For each year increase in the duration of diabetic follow up, the odds of experiencing ED will increase by 1.67 [AHR=1.67; 95\% CI: 1.40-2.00]. This finding is conformable with reports of previous studies conducted in China, [18] Israel, [28] Nigeria, [31] Africa, [38], and Northern Ethiopia [16]. The possible rationale could be when the number years on of diabetic follow-up increased, the tendency to develop diabetic complications such as nerve and blood vessel damage increased as well. Hence, these physiologic changes will be finally linked with decreased penile blood flow and the nerves that control erection resulting in reduced sexual performance and erectile dysfunction.

Furthermore, those patients who took oral hypoglycemic agents for DM management were also reported to be 6.27 times at an increased risk of experiencing ED compared to those who managed with insulin injection $[\mathrm{AHR}=6.27 ; 95 \% \mathrm{CI}$ : 2.01-19.58]. However, none of the previously conducted literature identified the type of diabetic medication as an independent factor for erectile dysfunction. Even though managing DM reduces diabetic complications by slowing down the damage to nerves and blood vessels, oral hypoglycemic agents mainly metformin, the most prescribed drug for diabetics in our settings, is associated with a significant reduction in testosterone levels. Consequently, this could lead to reduced sex drive and low-testosterone induced erectile dysfunction [39].

According to earlier studies carried out in Africa and Asia, factors such as advanced age, diabetic complications (including peripheral neuropathy, retinopathy, heart disease, and hypertension), type of DM, and depression were notified as independent predictors of erectile dysfunction $[18,25,28,29,32,34,39,40]$. Nevertheless, these factors were not found to have a statistically significant association with erectile dysfunction. This disparity might be due to the dissimilarity of characteristics of study subjects such that the median age of participants in the current study was relatively younger than previous studies [18,28,31]. Moreover, some of the former studies also included patients above the aged 30 and 40 years, which would magnify the role of age on the outcome interest $[29,40]$.

\section{Conclusion}

The prevalence of erectile dysfunction among male patients diagnosed with DM was found to be high in this study since it is higher than the global estimated range of 35-75\%. Moreover, diabetic patients who had prolonged diabetic follow-up, family monthly-income above the poverty line, used oral hypoglycemic agents for DM management, and those who did not actively engage in daily physical exercise were at high risk to experience erectile dysfunction.

\section{Acknowledgments}

Study participants, data collectors, supervisors, hospital staff, and administrators were appreciated for their unfailing contribution. The authors would also like to thank Aksum University that covers the financial requirements of this study.

\section{Funding}

This research received no specific grant from any funding agency in the public, commercial, or not-for-profit sectors. However, the financial backing of this research was covered by Aksum University. The funder had no role in study design, data collection, analysis, preparation of the manuscript, and decision to publish.

\section{Data sharing statement}

Extra data that support the findings of this study are available from the corresponding author upon reasonable request and can be shared upon legal request via bayayibignabez@gmail.com

\section{Authors' contributions}

All authors made a significant contribution to the work reported, whether that is in the conception, study design, execution, acquisition of data, analysis, and interpretation, or in all these areas; took part in drafting, revising, or critically reviewing the article; gave final approval of the version to be published; have agreed on the journal to which the article has been submitted; and agreed to be accountable for all aspects of the work.

\section{Ethical approval and consent to participate}

Ethical clearance and approval were obtained from Aksum University, College of Health Science institutional review board. Then officials at different levels of the selected hospitals had been 
communicated through a cooperation letter. The study was conducted per the declaration of Helsinki. The purpose of the study was explained to and written informed consent was obtained from men diabetic patients to confirm willingness. They were notified that they have the right to refuse or terminate the interview at any time. Confidentiality of the information was secured throughout the study process.

\section{Consent for publication}

Not applicable.

\section{Disclosure}

The authors declared no conflict of interest.

\section{References}

1. Ogurtsova K, da Rocha Fernandes J, Huang Y (2017) IDF Diabetes Atlas: Globa estimates for the prevalence of diabetes for 2015 and 2040. Diabetes Research and Clinical Practice 128: 40-50. [Crossref]

2. Campos C (2012) Chronic hyperglycemia and glucose toxicity: pathology and clinical sequelae. Postgraduate Medicine 124: 90-97.

3. Lu CC, Jiann BP, Sun CC, Lam HC, Chu CH (2009) Association of glycemic control with the risk of erectile dysfunction in men with type 2 diabetes. The Journal of Sexual Medicine 6: 1719-1728.

4. Rahman S, Rahman T, Ismail AAS, Rashid ARA (2007) Diabetes associated macro vasculopathy: pathophysiology and pathogenesis. Diabetes, Obesity, and Metabolism 9: 767-780.

5. Droller MJA (1993) NIH consensusdevelopment panel on impotence: impotence. JAMA 270: 83. [Crossref]

6. Idung AU, Abasiubong F, Ukott IA, Udoh SB, Unadike BC (2012) Prevalence and risk factors of erectile dysfunction in Niger delta region, Nigeria. African health sciences 12: $160-165$.

7. Selvin E, Burnett AL, Platz EA (2007) Prevalence and risk factors for erectile dysfunction in the US. The American Journal of Medicine 120: 151-157.

8. Sadock BJ, Sadock VA (2014) Kaplan and Sadock's synopsis of psychiatry: Behavioral sciences/clinical psychiatry. Ed: 11. Lippincott Williams \& Wilkins.

9. Esposito K, Ciotola M, Marfella R, Di Tommaso D, Cobellis L (2005) Sexual dysfunction in women with metabolic syndrome. Diabetes Care 28: 756-756.

10. Seftel AD, Sun P, Swindle R (2004) The prevalence of hypertension, hyperlipidemia, diabetes mellitus, and depression in men with erectile dysfunction. The Journal of urology 171: 2341-2345.

11. Unadike B, Eregie A, Ohwovoriole A (2008) Prevalence and types of sexual dysfunction among males with diabetes in Nigeria. Diabetes International 1: 18-20.

12. Ziaei-Rad M, Vahdaninia M, Montazeri A (2010) Sexual dysfunctions in patients with diabetes: a study from Iran. Reproductive biology and endocrinology: $R B \& E$ 18: 8:50.

13. Levy J (2002) Impotence and its medical and psychosocial correlates: results of the Massachusetts Male Aging Study. The British Journal of Diabetes \& Vascular Disease 2: $278-280$.

14. Dunn KM, Croft PR, Hackett GI (1999) Association of sexual problems with social, psychological, and physical problems in men and women: a cross-sectional population survey. Journal of Epidemiology \& Community Health 53: 144-148.

15. Kubin M, Wagner G, Fugl-Meyer AR (2003) Epidemiology of erectile dysfunction. International Journal of Impotence Research 15: 63.

16. Seid A, Gerensea H, Tarko S, Zenebe Y, Mezemir R (2017) Prevalence and determinants of erectile dysfunction among diabetic patients attending in hospitals of the central and northwestern zone of Tigray, northern Ethiopia: a cross-sectional study. BMC Endocrine Disorders 17: 16

17. Melnik T, Soares B, Nasello AnG (2007) Psychosocial interventions for erectile dysfunction. Cochrane Database of Systematic Reviews 3.

18. Yang G, Pan C, Lu J (2010) Prevalence of erectile dysfunction among Chinese men with type 2 diabetes mellitus. International Journal of Impotence Research 22: 310317.

19. Tigray Regional Health Bureau (2018) Regional estimates of the population with a conversion factor. Unpublished resources: Tigray Regional Health Bureau.
20. Rosen RC, Cappelleri J, Smith M, Lipsky J, Pena B (1999) Development and evaluation of an abridged, 5-item version of the International Index of Erectile Function (IIEF5 ) as a diagnostic tool for erectile dysfunction. International Journal of Impotence Research 11: 319

21. Abiola T, Udofia O, Zakari M (2013) Psychometric properties of the 3-item Oslo socia support scale among clinical students of Bayero University Kano, Nigeria. Malaysian Journal of Psychiatry 22: 32-41.

22. Reda AA (2011) Reliability and validity of the Ethiopian version of the hospital anxiety and depression scale (HADS) in HIV infected patients. PLoS One 6: e16049.

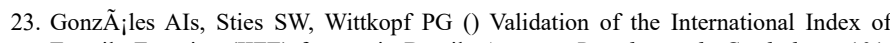
Erectile Function (IIFE) for use in Brazil. Arquivos Brasileiros de Cardiologia 101: 176-182.

24. WHO (1995) Physical status: the use and interpretation of anthropometry: report of a WHO Expert Committee: WHO Technical Report. Geneva: WHO.

25. Walle B, Lebeta KR, Fita YD, Abdissa HG (2018) Prevalence of erectile dysfunction and associated factors among diabetic men attending the diabetic clinic at Felege Hiwo Referral Hospital, Bahir Dar, North West Ethiopia, 2016. BMC Res Notes 11: 130.

26. Michael Dansinger (2020) Erectile dysfunction and diabetes. WebMed. Available at www.webmed.com/erectile-dysfunction/guide/ed-diabetes.

27. Alana Biggers, Goretti Cowler (2020) Does diabetes cause erectile dysfunction? Medical news today.

28. Roth A, KalterâLftbovhx O, Kerbis Y (2003) Prevalence and risk factors for erectile dysfunction in men with diabetes, hypertension, or both diseases: a community survey among 1,412 Israeli men. Clinical Cardiology: An International Indexed and Peera Reviewed Journal for Advances in the Treatment of Cardiovascular Disease 26: 25-30.

29. Sharifi F, Asghari M, Jaberi Y, Salehi O, Mirzamohammadi F (2012) Independent predictors of erectile dysfunction in type 2 diabetes mellitus: Is it true what they say about risk factors? ISRN Endocrinol 20: 502353-502353.

30. Atosona A, Larbie C, Tandoh MA, Nsiah K (2016) Prevalence, risk factors, and predictors of diabetes-related sexual dysfunction. JAMMR 17: 11

31. Theophilus Ugwu, Ignatius Ezeani, Samuel Onung, Babatope Kolawole, Ikem R (2016) Predictors of erectile dysfunction in men with type 2 diabetes mellitus referred to a tertiary healthcare centre. Advances in Endocrinology 2.

32. Mutagaywa RK, Lutale J, Aboud M, Kamala BA (2014) Prevalence of erectile dysfunction and associated factors among diabetic men attending diabetic clinic at Muhimbili National Hospital in Dar-es-Salaam, Tanzania. The Pan African Medical Journal 17: 227.

33. Ungaya GML (2011) The prevalence of sexual dysfunction among patients with diabetes mellitus attending the outpatient diabetic clinic at kenyatta national hospital. University of Nairobi.

34. Asefa A, Nigussie T, Henok A, Mamo Y (2019) Prevalence of sexual dysfunction and related factors among diabetes mellitus patients in Southwest Ethiopia. BMC Endocr Disord 19: 141.

35. Martyn A, Eric A (2014) Erectile dysfunction in the patient with diabetes mellitus $A J M C 4$.

36. David FP, Wessells H (2017) Erectile dysfunction in diabetic patients. Diabetes Spectrum 17.

37. Beverley Bostock-Cox (2017) Understanding the link between obesity and diabetes. Nursing standards England 7.

38. Shiferaw WS, Akalu TY, Petrucka PM, Areri HA, Aynalem YA (2020) Risk factors of erectile dysfunction among diabetes patients in Africa: A systematic review and metaanalysis. J Clin Transl Endocrinol 21: 100232.

39. Al-Kuraishy HM, Al-Gareeb AI (2016) Erectile dysfunction and low sex drive in men with type 2 DM: The potential role of diabetic pharmacotherapy. J Clin Diagn Res 10: 21-26.

40. Kemp T, Rheeder P (2015) The prevalence and associations of erectile dysfunction in a South African male diabetic urban population. Journal of Endocrinology, Metabolism, and Diabetes of South Africa 20: 134-139.

Copyright: $(\mathrm{C} 2020$ Gebreegziabhier G. This is an open-access article distributed under the terms of the Creative Commons Attribution License, which permits unrestricted use, distribution, and reproduction in any medium, provided the original author and source are credited. 